\title{
Article
}

\section{Implants versus Lipograft - analysis of long-term results after congenital breast asymmetry procedure}

\author{
Vanessa Brébant 1,*, Maximilian Weiherer ${ }^{2}$, Vivien Noisser ${ }^{1}$, Stephan Seitz ${ }^{3}$, Lukas Prantl ${ }^{1}$, Andreas \\ Eigenberger ${ }^{1,4}$ \\ 1 University Center for Plastic, Aesthetic, Hand and Reconstructive Surgery, Regensburg, Germany \\ 2 Regensburg Medical Image Computing (ReMIC), Ostbayerische Technische Hochschule Regensburg \\ (OTH Regensburg), Germany \\ 3 Department of Obstetrics and Gynecology, Caritas Hospital St. Josef, University of Regensburg, Germany \\ 4 Faculty of Mechanical Engineering, Ostbayerische Technische Hochschule Regensburg (OTH Regensburg), \\ Germany \\ * Correspondence: vanessa.brebant@ukr.de, Tel.: +499417823110
}

\begin{abstract}
Congenital breast asymmetry represents a particular challenge to the classical techniques of plastic surgery due to a young group of patients. This study compares traditional breast augmentation using silicone implants to the more innovative lipograft technique regarding longterm results. To achieve this, we not only captured subjective parameters like satisfaction with outcome and symmetry, but also objective parameters such as breast volume and anthropometric measurements. Objective examination was performed manually and by using the Vectra ${ }^{\circledR} \mathrm{H} 2$ photogrammetry scanning system. Patients who underwent implant augmentation and lipograft both showed no significant differences in patient's satisfaction with surgical outcome $(p=0.55)$ and symmetry $(p=0.69)$. Furthermore, a breast symmetry of $93 \%$ in both groups was reported. Likewise, no statistically significant volume difference between left and right breast was observed in both groups $(p<0.41)$. However, on average, lipograft patients needed 1.3 procedures more until the desired result was achieved. In contrast, patients treated with implant-based breast augmentation usually need several implant changes during their life. In conclusion, both methods should be considered for patients with congenital breast asymmetry.
\end{abstract}

Keywords: congenital breast asymmetry; PROM, autologous fat injections, fat grafting, lipograft, implant augmentation, breast augmentation, $3 \mathrm{D}$ volumetry, three-dimensional imaging, Breast- $\mathrm{Q}^{\mathrm{TM}}$

\section{Introduction}

Severe asymmetry is one of the most common reasons for breast augmentation in patients under the age of 18 [1]. Especially congenital breast asymmetry affects young women in puberty wherefore the patients often face a corrective surgery at a young age. As a result, the long-term outcome of the surgical treatment is crucial.

Implant augmentation is the standard procedure for treatment of asymmetry, volume deficits, or macromastia [2]. For patients with congenital breast asymmetry, implant augmentation is usually associated with several implant changes in the course of their lives. Hence, surgical procedures are required providing better long-term stability. Recent, promising techniques are based on autologous fat transfers, with lipograft being one of them. In previous work, we could prove that enriched autologous fat grafting offers good long-term results in patients with midface deficiency, improving facial volume loss and skin quality.[3]

In this study, we examine long-term outcomes for patients with congenital breast asymmetry that underwent lipograft or silicone implant augmentation. Since both surgical methods are described differently in literature, we present our protocols used below. Regarding the long-lasting result, both, patient satisfaction and objective parameters such as postoperative volume difference and symmetry are analysed. We 
hypothesize that breast augmentation with lipograft offers at least as equally objective and subjective long-term results as breast augmentation with silicone implants.

\section{Materials and Methods}

Study design and patients

A total of 32 patients with corrected breast asymmetry, either by lipograft or silicone implant, were included in our retrospective cohort study. Out of the 32 patients, 16 underwent alloplastic and 16 autologous breast augmentation $(n=16)$. On average, patients were examined $7 \pm 3.2$ years after surgery. Inclusion criterion was any kind of congenital breast asymmetry operated in our institution from January 2008 until December 2019. Patients who were treated with both, lipograft and silicone implant or missed follow-up examinations were excluded. The data collection period was from March 2020 to July 2020 and had been approved by the Ethics Committee of the University Hospital of Regensburg (20-1654-101). Relevant metadata are shown in Table 1.

Table 1. Comparison of the alloplastic (Implant) and the autologous (Lipograft) group.

\begin{tabular}{|c|c|c|c|c|}
\hline & \multicolumn{2}{|c|}{ Implant (n=16) } & \multicolumn{2}{|c|}{ Lipograft $(n=16)$} \\
\hline & Mean $( \pm S D)$ & Range & Mean $( \pm S D)$ & Range \\
\hline Age $^{*}$ [years] & $21( \pm 4.9)$ & $16-33$ & $20( \pm 3.7)$ & $16-29$ \\
\hline BMI $\left[\mathrm{kg} / \mathrm{m}^{2}\right]^{* *}$ & $21.9( \pm 2.1)$ & $18-26$ & $24.4( \pm 3.8)$ & $20-33$ \\
\hline Postoperative [years] & $7.2( \pm 3.6)$ & $2.6-12$ & $6.5( \pm 2.8)$ & $0.9-10.2$ \\
\hline Cup size & - & B -E & - & $A-E$ \\
\hline Number of operations & $1.6( \pm 0.6)$ & $1-3$ & $2.9( \pm 1.3)$ & $1-5$ \\
\hline
\end{tabular}

*at the time of first breast surgery

** at the time of last breast surgery

\section{Lipograft}

Lipograft mainly consists of a harvesting, processing, and injection step. Following the S2K guideline, sodium chloride $(0.9 \%)$ and adrenaline $(1: 200,000)$ was infiltrated 15 minutes before harvesting. According to the study of Sommer [4], the cell vitality of the harvested fat graft is equal in every harvesting site. Hence, the harvesting areas were chosen according to the available volume of fat and patient desires. Harvesting was performed using waterjet-assisted liposuction (HumanMed BodyJet, Schwerin, Germany) and harvesting cannulas. The harvested adipose tissue is composed of adipocytes and stromal vascular fraction cells, including adipose-derived stem cells (ASCs), preadipocytes, fibroblasts, vascular endothelial cells, and a variety of immune cells [5].

During the processing step, as many blood, serum, and tumescent solution as possible was separated from vital mature adipocytes and adipose progenitor cells. This was performed using sedimentation or centrifugation (3,000 rpm, $2 \mathrm{~min})$. After discarding the unneeded parts, the processed fat is transplanted as uniformly as possible through multiple $2 \mathrm{~mm}$ stab incisions using LuerLock syringes. On average, $502 \pm 337 \mathrm{ml}$ lipograft were injected in each lipograft patient.

Implant augmentation

With over 1.8 million surgeries each year, implant-based breast augmentation is the most common aesthetic surgical procedure worldwide. Depending on the individual 
subcutaneous fat layer, implants are inserted either subpectorally or subglandularly. Out of our 16 patients who underwent breast augmentation with silicone implants, seven patient's implants were placed subglandular and nine subpectoral. In order to achieve a natural looking result, scaring should be hidden in the submammary fold. On average, implants with $235.6 \mathrm{ml}$ were chosen for augmentation (ranging from $150 \mathrm{ml}$ to $355 \mathrm{ml}$ ). We could show in a recent study, that textured breast implants cause a topographic change, particularly on the upper breast, the décolleté [6].

Objective outcome measurements

In the long-term follow-up, surgical results were evaluated with two objective outcome parameters: symmetry and volume difference between both breasts. Therefore, all patients were measured manually using a classic tape along the skin surface. Breast symmetry was quantified using the Symmetry Index (SI). According to [7], the SI allows a symmetry calculation between 0 (worst) and 1 (best) by means of seven anthropomorphic measurements, shown in Figure 1.

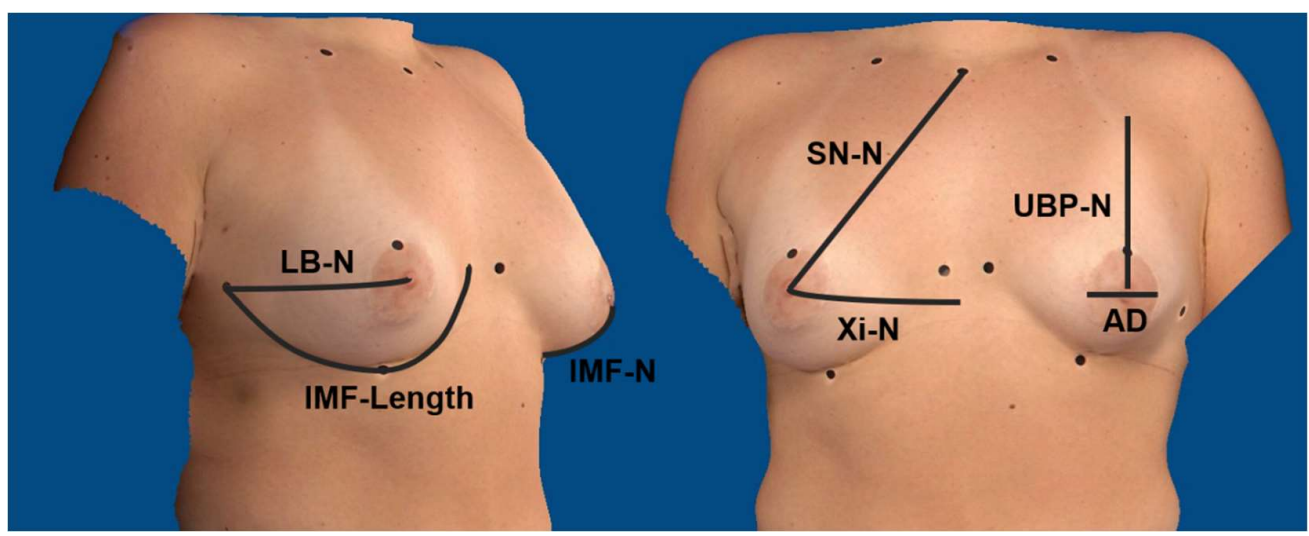

Figure 1. The anthropometric measurements used for SI calculation: LB-N: Lateral Breast Pole to Nipple, IMF-Length: Inframammary Fold Length, IMF-N: Inframammary Fold to Nipple, SN-N: Sternal Notch to Nipple, Xi-N: Xiphoid to Nipple, UBP-N: Upper Breast Pole to Nipple, AD: Areola diameter.

Post-operative breast volume was calculated using the Breast-V formula as proposed by Longo et al. [8] for ptotic and extended by Huang et al. [9] for non-ptotic breasts. Both formula had been used in several studies[10-13] Additionally, we measured breast volumes and various breast dimensions using the portable Vectra ${ }^{\circledR}$ H2 (Canfield Scientific, USA) photogrammetry scanning system. This system allows to reproduce the patient's total surface information. It comes with Vectra ${ }^{\circledR}$ Breast-Sculptor ${ }^{\mathrm{TM}}$ (Canfield Scientific,USA) software for point cloud processing and mesh generation. Using this software, the 3D model can be analysed in terms of breast volume and various breast dimensions as demonstrated in several studies[14-22]. Therefore, thirteen points were marked at the patient and subsequently picked in Breast-Sculptor ${ }^{\mathrm{TM}}$, see Figure 2. 


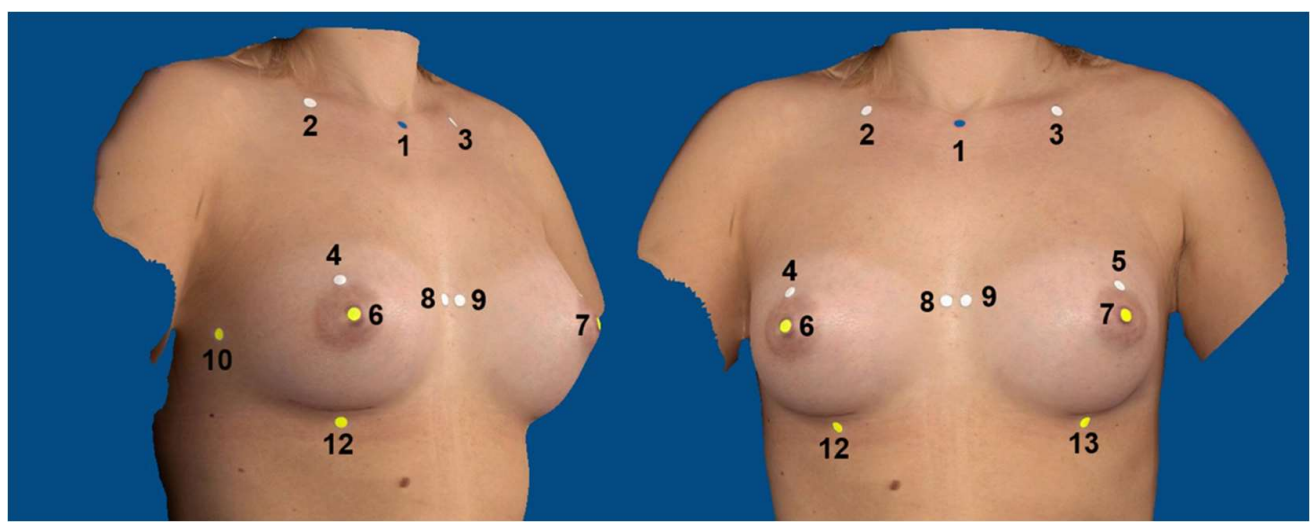

Figure 2. Reference points for Canfield Vectra ${ }^{\circledR} \mathrm{H} 2$ (picture created with Canfield Vectra ${ }^{\circledR} \mathrm{H} 2$ and edited), 1: sternal notch, 2-3: centre of the clavicle, 4-5: most cranial point of the areola, 6-7: nipple, 8-9: end of medial inframammary fold, 10-11: end of lateral inframammary fold, 12-13: most caudal point of the inframammary fold.

Patient-related outcome measures

To measure the patient-related outcome, we utilized the Breast- $\mathrm{Q}^{\mathrm{TM}}$ questionnaire (Breast- $\mathrm{Q}^{\mathrm{TM}}$ Version $2.0^{\circ}$, Augmentation Modules Pre- and Postoperative Scales, The University of British Columbia, licensed for non-profit users by Memorial Sloan Kettering Cancer Center and translated by Mapi Research Trust, 2008). This validated questionnaire is commonly used in similar studies to request patient's satisfaction after breast augmentation $[23,24]$. The Breast- $\mathrm{Q}^{\mathrm{TM}}$ shows the satisfaction in percent, with $0 \%$ being the worst and $100 \%$ the best possible value. In addition to the Breast- $\mathrm{Q}^{\mathrm{TM}}$, the satisfaction with breast symmetry was questioned retrospectively using a scale from 1 (worst) to 4 (best).

\section{Statistical analysis}

SPSS $^{\circledast}$ Statistics Version 25.0.0. (IBM ${ }^{\circledR}$, Armonk; New York) was used to perform statistical analysis. Since our data did not follow a normal distribution (KolmogorovSmirnov test and the Shiparo-Wilk test) in both groups and in any of the cases, we applied the Mann-Whitney test to detect differences between the groups. P-values $<0.05$ were considered as statistically significant.

\section{Results}

For all 32 patients included, the satisfaction with surgical outcome, the SI, and the volume difference was collected. Exemplary, Figure 3 and Figure 4 show a pre- and postoperative comparison for both surgical methods.
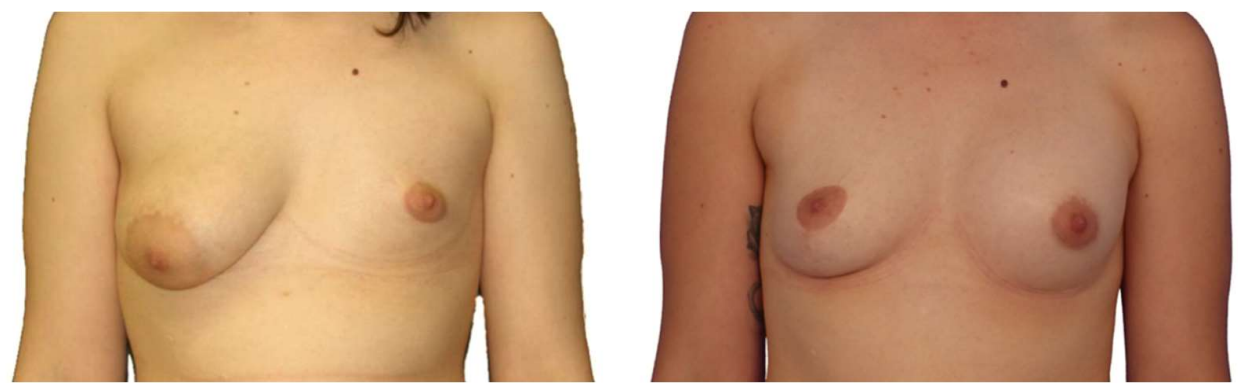
Figure 3. Patient with congenital breast asymmetry and tubular deformity. Left: preoperative 2017.Right: postoperative, 2020, after unilateral breast augmentation with silicone implant and contralateral mastopexy.
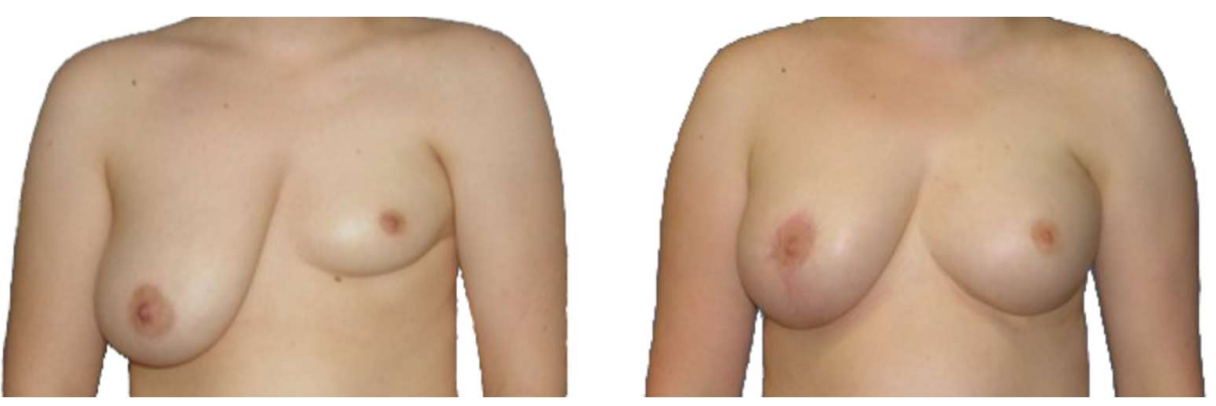

Figure 4. Patient with breast asymmetry and Poland's syndrome. Left: preoperative, before lipograft therapy in two sessions (surgery in 2010 and 2013). Right: postoperative 2020, after contralateral mastopexy.

\subsection{Patient's satisfaction with surgical intervention}

On average, a satisfaction with surgical outcome of $71 \pm 27 \%$ was reported for patients treated with lipograft. Patients who underwent implant augmentation were satisfied by $76 \pm 19 \%$ (Figure 5). The Mann-Whitney test revealed no significant difference $(\mathrm{p}=0.554)$.

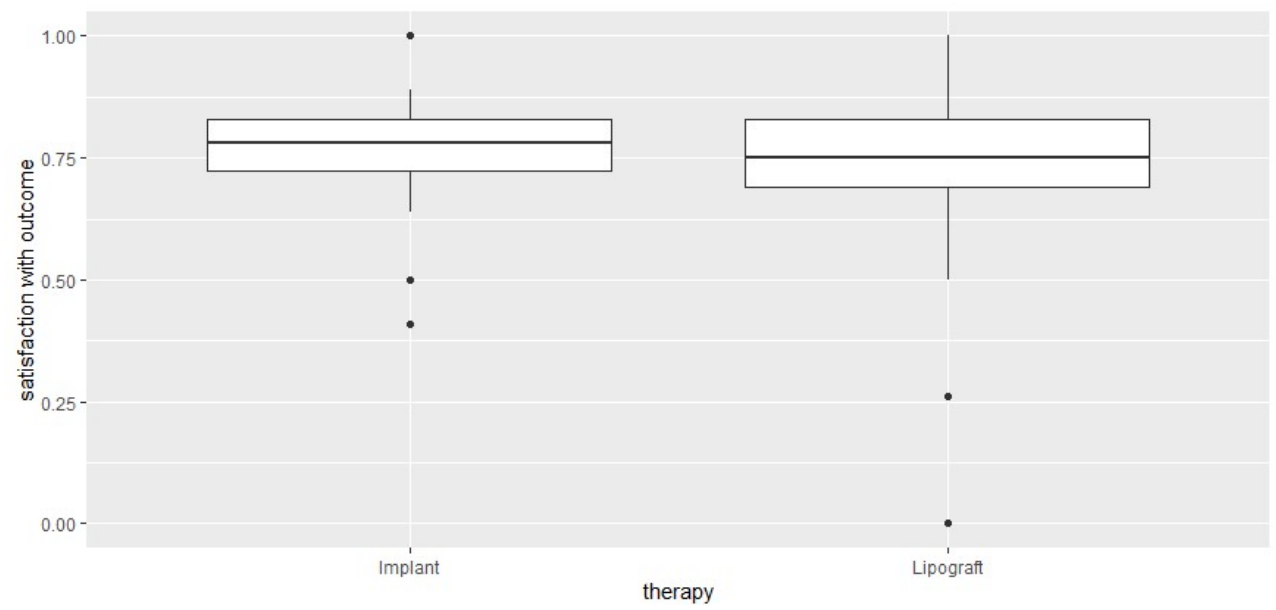

Figure 5. Boxplot of the patient satisfaction after both therapies, evaluated using the Breast- $\mathrm{Q}^{\mathrm{TM}}$ questionnaire.

The additional question about satisfaction with breast symmetry (on a scale from 1 to 4 ) was answered with $2.7 \pm 1.1$ for implant augmentation and $2.6 \pm 1.0$ for lipograft. The difference between both methods which is shown in Figure 6 is not significant $(p=0.69)$. 


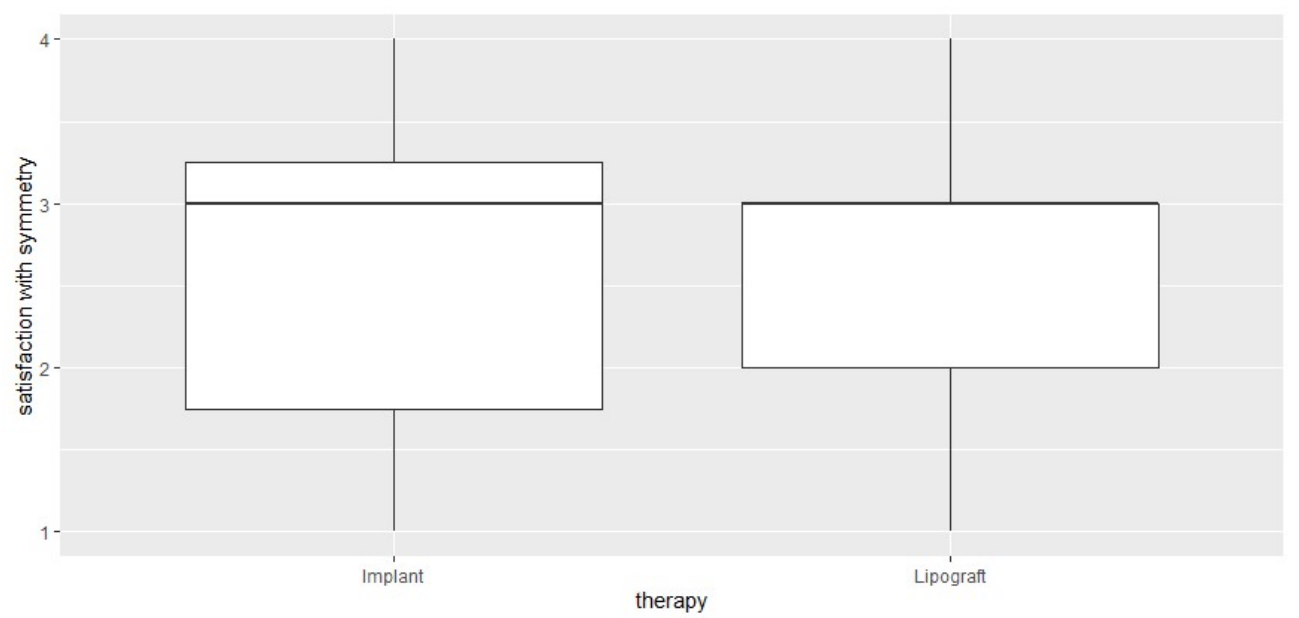

Figure 6. Boxplot of the patient satisfaction with breast symmetry, evaluated using a scale from 1 (worst) to 4 (best).

\subsection{Objective outcome evaluation}

We evaluated the SI and the volume difference between both breasts postoperatively. As shown in Figure 7, the SI is $93 \pm 3 \%$ for lipograft and $93 \pm 5 \%$ for implant augmentation. The difference between both methods is not statistically significant (p-value: 0.762 ).

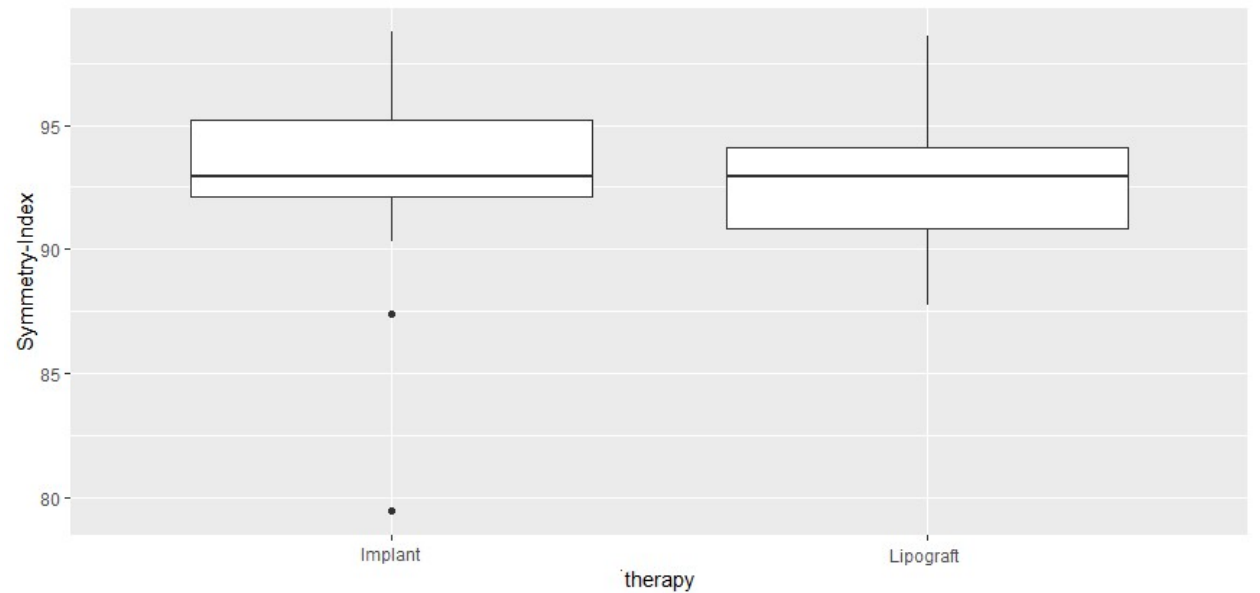

Figure 7. Boxplot comparing the SI calculated on patients treated with implant augmentation and lipograft.

By means of the Breast- $V$ formula, a postoperative volume difference of $27 \pm 30 \mathrm{~g}$ for implant augmentation and $50 \pm 47 \mathrm{~g}$ for lipograft was calculated. In contrast, using Vectra $^{\circledR}$, a postoperative volume difference of $74 \pm 87 \mathrm{ml}$ for implant augmentation and $86 \pm$ $70 \mathrm{ml}$ for lipograft was measured. Neither the data collected with Vectra ${ }^{\circledR}$ nor the Breast$\mathrm{V}$ were statistic significant (p-value 0.41 and 0.13 ).

The difference in volume is assessed differently depending on the total volume of the breast. Therefore, the volume difference was set in relation to the volume of the bigger breast. The results are shown in Figures 8 and 9. 


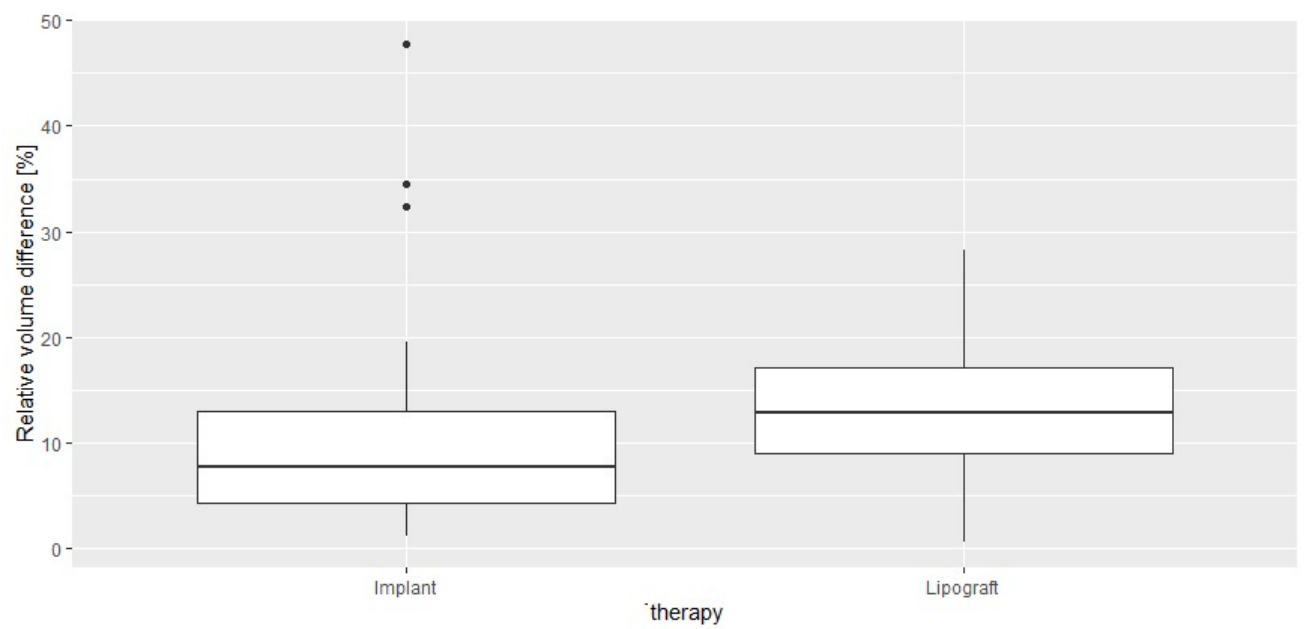

Figure 8. Comparison of the postoperative, relative volume difference measured using the 3D-scanning system Vectra ${ }^{\circledR}$.

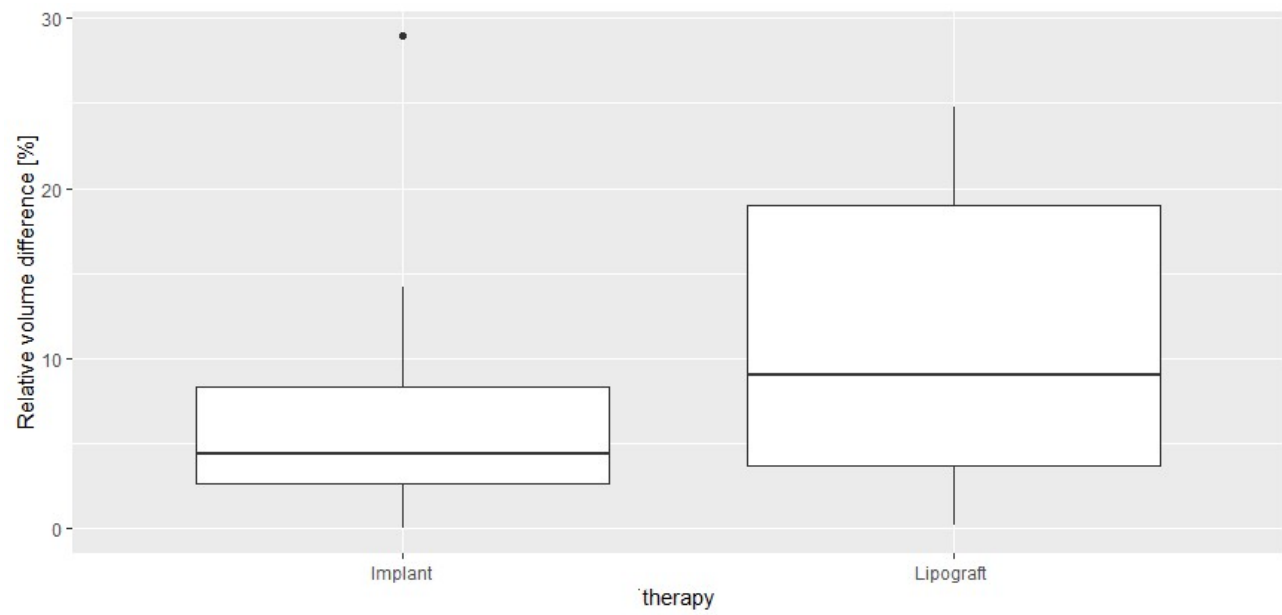

Figure 9. Comparison of the postoperative, relative volume difference measured using the Breast- $\mathrm{V}$ formula.

\section{Discussion}

In this study, we compared silicone implants and lipograft as possible therapies correcting congenital breast asymmetry. Since the time of operation is usually early in the patient's life, satisfaction with results over many years is decisive. Therefore, our collective of patients was postoperative for 6.8 years, on average.

To measure the patient-related outcome on a long-term basis we utilized the Breast$\mathrm{Q}^{\mathrm{TM}}$ questionnaire.

Lipograft and implant therapy yielded similarly good results (average subjective long-term outcome satisfaction of $74 \%$ ). We thus consider both therapies as current and relevant. This is also confirmed by a study conducted by Sandsmark et al. [25], in which no significant difference between therapy with or without implants was found. Additionally, our study supports the results of Kuzbari et al. [26] as well. Although their study had a different focus and did not take lipograft into account, they also reported of long-term satisfaction with correction of congenital breast asymmetry, and general patient satisfaction with long-term outcome.

As a drawback of the Breast- $\mathrm{Q}^{\mathrm{TM}}$, the satisfaction with symmetry after surgery is not asked in the questionnaire. Hence, we added an additional question, which showed equal 
results for both surgical methods. Although the data showed a large deviation, on average, the patients were satisfied with their long-term breast symmetry. This is consistent with our objective results on symmetry, as our newly developed Symmetry Index showed good results for both methods (93\%). The Department of Plastic Surgery at The University of Texas MD Anderson Cancer Center, Houston found that a substantial portion of women (50.6\% of a non-operated cohort) exhibit a volume difference greater than $50 \mathrm{ml}$ between right and left breast [27]. This study provides normative data on the extent of breast asymmetry in preoperative patients that can guide us in setting realistic goals for reconstruction procedures. Indeed, our postoperative cases show a symmetry near to reality.

However, some patients reported postoperative numbness or hypersensitivity. This was also observed in other studies including Heine et al. [28] who described better sensitivity of the breast after lipograft than after implant reconstruction. As previously described, the objective assessment of breast sensitivity is of great interest and should be evaluated in a follow-up work [28]

Anthropometric measurements utilised in the Breast- $\mathrm{V}$ formula provide a valid approximation of the breast volume [9]. However, three-dimensional imaging with Vectra ${ }^{\circledR}$ in combination with Breast-Sculptor ${ }^{\mathrm{TM}}$ (Canfield Scientific, USA) is a validated method for calculating breast volumes as well. Indeed, in our study, we could show a good correlation of breast volume differences between the Breast- $V$ approximation and the three-dimensional-based calculation.

Of course, three-dimensional imaging is superior to anthropometric measurements as it creates a digital twin and,therefore, allows for further comparisons without the need of an additional, physical examination. This makes it possible to analyse even small volume deficits or scars, which are not represented by anthropomorphic measurements.

The oncogenic effect of fat grafting remains controversial [29,30]. A retrospective study published in 2016 [31], which included 719 patients with benign and malignant breast disease and fat grafting showed no evidence of an increased incidence of locoregional recurrence, systemic recurrence, or new onset of breast carcinoma. Since breast augmentation with silicone implants presents long-term safety concerns (ALCL, capsular fibrosis, leakage) and will lead to implant changes, it could be advantageous to operate young patients with congenital breast asymmetry using lipograft. In addition, unilateral breast augmentation with silicone implants leaves a texture difference and an asymmetry in the palpation findings.

Lipograft for breast augmentation required on average 2.9 sessions to achieve the desired result, which is significantly more than in the implant group (1.6). As mentioned before, patients with congenital breast asymmetry are usually operated very early in their life, leading to several implant changes. Hence, compared to lipograft, the number of operations required for implant augmentation is expected to increase due the patient's total lifespan. Furthermore, studies showed that lipograft can be enriched with progenitor and stem cells using mechanical shear stress only, without causing a manipulation of the cells secretome [32]This could improve the of adipose cell transplantation and strengthen the patient's satisfaction with autologous fat transfer.

A limitation of this study is its small sample size. Although the sample size is common for congenital breast asymmetry studies [26][33][34] and both of our groups are very similar in terms of age and BMI, our collective size of 32 allows only limited generalisations. In the future, a multicentre approach may generate interesting results considering improvements in lipograft processing and lighter implants.

\section{Conclusions}

In this study, a non-significant difference in the long-term outcome between lipograft and silicone implant augmentation could be shown, either subjectively or objectively. 
Both surgical procedures showed a good satisfaction with surgical outcome and breast symmetry. Lipograft needed more sessions to achieve the desired result, but implant augmentation led to several implant changes in this young patient population.

On the one hand, the tissue compatibility of breast implants may improve in the future and thus require fewer implant changes. On the other hand, the take of adipose cell transplantation may improve as well, so that fewer surgical sessions will be necessary. Therefore, prospective studies and investigations are further needed to compare competitive surgical techniques of breast augmentation in congenital malformations.

Author Contributions: Conceptualization, V.B., L.P. and V.N.; methodology, V.N; software, A.E.; validation, M.W., L.P. and A.E.; formal analysis, A.E., V.B.; investigation, V.N., V.B.; writing-original draft preparation, V.B.; writing - review and editing, A.E, V.N., M.W, S.S..; visualization, M.W.; supervision, L.P, S.S.; project administration, V.B.; All authors have read and agreed to the published version of the manuscript.

Funding: This research received no external funding.

Institutional Review Board Statement: The study was conducted according to the guidelines of the Declaration of Helsinki and approved by the Ethics Committee of the University of Regensburg, Germany (Approval number: 20-1654-101).

Informed Consent Statement: Informed consent was obtained from all subjects involved in the study. Written informed consent has been obtained from the patients to publish this paper.

Data Availability Statement: Data sharing is not applicable to this article.

Conflicts of Interest: The authors declare no conflict of interest.

\section{References}

1. Isaps Global Survey Results 2018 Available online: https://www.isaps.org/wp-content/uploads/2019/12/ISAPS-Global-SurveyResults-2018-new.pdf (accessed on 8 July 2021).

2. Vanessa Brebant; Lukas Prantl Die Korrektur von kongenitalen Brustfehlbildungen durch autologe Fetttransplantation. In Ästhetische Chirurgie von Heimburg, Richter, Lemperle; ecomed Medizin, 2019.

3. Prantl, L.; Brix, E.; Kempa, S.; Felthaus, O.; Eigenberger, A.; Brébant, V.; Anker, A.; Strauss, C. Facial Rejuvenation with Concentrated Lipograft-A 12 Month Follow-Up Study. Cells 2021, 10, doi:10.3390/cells10030594.

4. Sommer, B.; Sattler, G. Current Concepts of Fat Graft Survival: Histology of Aspirated Adipose Tissue and Review of the Literature. Dermatol Surg 2000, 26, 1159-1166.

5. Hanke, A.; Prantl, L.; Wenzel, C.; Nerlich, M.; Brockhoff, G.; Loibl, M.; Gehmert, S. Semi-Automated Extraction and Characterization of Stromal Vascular Fraction Using a New Medical Device. Clin Hemorheol Microcirc 2016, 64, 403-412, doi:10.3233/CH-168124.

6. Lotter, L.; Brébant, V.; Eigenberger, A.; Hartmann, R.; Mueller, K.; Baringer, M.; Prantl, L.; Schiltz, D. “Topographic Shift”: A New Digital Approach to Evaluating Topographic Changes of the Female Breast. Arch Gynecol Obstet 2021, 303, 515-520, doi:10.1007/s00404-020-05837-3.

7. Hartmann, R.; Weiherer, M.; Schiltz, D.; Seitz, S.; Lotter, L.; Anker, A.; Palm, C.; Prantl, L.; Brébant, V. A Novel Method of Outcome Assessment in Breast Reconstruction Surgery: Comparison of Autologous and Alloplastic Techniques Using ThreeDimensional Surface Imaging. Aesth Plast Surg 2020, doi:10.1007/s00266-020-01749-4.

8. Longo, B.; Farcomeni, A.; Ferri, G.; Campanale, A.; Sorotos, M.; Santanelli, F. The BREAST-V: A Unifying Predictive Formula for Volume Assessment in Small, Medium, and Large Breasts. Plastic and Reconstructive Surgery 2013, 132, 1e-7e, doi:10.1097/PRS.0b013e318290f6bd.

9. Huang, N.; Quan, C.; Mo, M.; Chen, J.; Yang, B.; Huang, X.; Wu, J. A Prospective Study of Breast Anthropomorphic Measurements, Volume and Ptosis in 605 Asian Patients with Breast Cancer or Benign Breast Disease. PLoS One 2017, 12, doi:10.1371/journal.pone.0172122.

10. Catanuto, G.; Rocco, N.; Nava, M.B. Surgical Decision Making in Conservative Mastectomies. Gland Surg 2016, 5, 69-74, doi:10.3978/j.issn.2227-684X.2015.05.12.

11. Rancati, A.; Angrigiani, C.; Hammond, D.; Nava, M.; Gonzalez, E.; Rostagno, R.; Gercovich, G. Preoperative Digital Mammography Imaging in Conservative Mastectomy and Immediate Reconstruction. Gland Surg 2016, 5, 9-14, doi:10.3978/j.issn.2227-684X.2015.08.01. 
12. Ji, K.; Luan, J.; Liu, C.; Mu, D.; Mu, L.; Xin, M.; Sun, J.; Yin, S.; Chen, L. A Prospective Study of Breast Dynamic Morphological Changes after Dual-Plane Augmentation Mammaplasty with 3D Scanning Technique. PLoS One 2014, 9, doi:10.1371/journal.pone.0093010.

13. Atici, Y.; Polat, B.; Erdogan, S.; Gürpınar, T.; Demiröz, S. Can Breast Asymmetry Following the Treatment of Juvenile Idiopathic Scoliosis with Growing Rod Be Prevented?: A Preliminary Analysis. J Korean Neurosurg Soc 2020, 63, 228-236, doi:10.3340/jkns.2019.0192.

14. Chae, M.P.; Rozen, W.M.; Spychal, R.T.; Hunter-Smith, D.J. Breast Volumetric Analysis for Aesthetic Planning in Breast Reconstruction: A Literature Review of Techniques. Gland Surg 2016, 5, 212-226, doi:10.3978/j.issn.2227-684X.2015.10.03.

15. Wesselius, T.S.; Verhulst, A.C.; Vreeken, R.D.; Xi, T.; Maal, T.J.J.; Ulrich, D.J.O. Accuracy of Three Software Applications for Breast Volume Calculations from Three-Dimensional Surface Images. Plastic and Reconstructive Surgery 2018, 142, 858-865, doi:10.1097/PRS.0000000000004728.

16. O'Connell, R.L.; Khabra, K.; Bamber, J.C.; deSouza, N.; Meybodi, F.; Barry, P.A.; Rusby, J.E. Validation of the Vectra XT ThreeDimensional Imaging System for Measuring Breast Volume and Symmetry Following Oncological Reconstruction. Breast Cancer Res Treat 2018, 171, 391-398, doi:10.1007/s10549-018-4843-6.

17. Koban, K.C.; Frank, K.; Etzel, L.; Schenck, T.L.; Giunta, R.E. 3D Mammometric Changes in the Treatment of Idiopathic Gynecomastia. Aesth Plast Surg 2019, 43, 616-624, doi:10.1007/s00266-019-01341-5.

18. Koban, K.C.; Schenck, T.; Metz, P.M.; Volkmer, E.; Haertnagl, F.; Titze, V.; Giunta, R.E. Auf dem Weg zur objektiven Evaluation von Form, Volumen und Symmetrie in der Plastischen Chirurgie mittels intraoperativer 3D Scans. Handchir Mikrochir plast Chir 2016, 48, 78-84, doi:10.1055/s-0042-104506.

19. Schenck, T.L.; Koban, K.C.; Giunta, R.E. Ermittlung objektiver 3-D Daten zur korrekten Planung von Folgeeingriffen nach Brustrekonstruktion. In Proceedings of the Senologie - Zeitschrift für Mammadiagnostik und -therapie; Georg Thieme Verlag KG, June 2018; Vol. 15, p. 124.

20. Diaz, J.F. Review of 494 Consecutive Breast Augmentation Patients: System to Improve Patient Outcomes and Satisfaction. Plast Reconstr Surg Glob Open 2017, 5, doi:10.1097/GOX.0000000000001526.

21. Camison, L.; Bykowski, M.; Lee, W.W.; Carlson, J.C.; Roosenboom, J.; Goldstein, J.A.; Losee, J.E.; Weinberg, S.M. Validation of the Vectra H1 Portable Three-Dimensional Photogrammetry System for Facial Imaging. Int J Oral Maxillofac Surg 2018, 47, 403410, doi:10.1016/j.ijom.2017.08.008.

22. Hartmann, R.; Weiherer, M.; Schiltz, D.; Baringer, M.; Noisser, V.; Hösl, V.; Eigenberger, A.; Seitz, S.; Palm, C.; Prantl, L.; et al. New Aspects in Digital Breast Assessment: Further Refinement of a Method for Automated Digital Anthropometry. Arch Gynecol Obstet 2020, doi:10.1007/s00404-020-05862-2.

23. Pusic, A.; Klassen, A.; Scott, A.; Klok, J.; Cordeiro, P.; Cano, S. Development of a New Patient-Reported Outcome Measure for Breast Surgery: The BREAST-Q. Plastic and Reconstructive Surgery 2009, 124, 345-353, doi:10.1097/PRS.0b013e3181aee807.

24. Cohen, W.A.; Mundy, L.R.; Ballard, T.N.S.; Klassen, A.; Cano, S.J.; Browne, J.; Pusic, A.L. The BREAST-Q in Surgical Research: A Review of the Literature 2009-2015. J Plast Reconstr Aesthet Surg 2016, 69, 149-162, doi:10.1016/j.bjps.2015.11.013.

25. Sandsmark, M.; Amland, P.F.; Samdal, F.; Skolleborg, K.; Åbyholm, F. Clinical Results in 87 Patients Treated for Asymmetrical Breasts: A Follow-up Study. Scandinavian Journal of Plastic and Reconstructive Surgery and Hand Surgery 1992, 26, 321-326, doi:10.3109/02844319209015278.

26. Kuzbari, R.; Deutinger, M.; Todoroff, B.P.; Schneider, B.; Freilinger, G. Surgical Treatment of Developmental Asymmetry of the Breast Long Term Results. Scandinavian Journal of Plastic and Reconstructive Surgery and Hand Surgery 1993, 27, 203-207, doi:10.3109/02844319309078112.

27. Cheong, A.L.; Liu, J.; Reece, G.P.; Nicklaus, K.M.; Catherine Bordes, M.; Hanson, S.E.; Markey, M.K.; Merchant, F.A. Natural Breast Symmetry in Preoperative Breast Cancer Patients. Plast Reconstr Surg Glob Open 2019, 7, e2297, doi:10.1097/GOX.0000000000002297.

28. Noisser, V.; Eigenberger, A.; Weiherer, M.; Seitz, S.; Prantl, L.; Brébant, V. “Surgery of Congenital Breast Asymmetry - Which Objective Parameter Influences the Subjective Satisfaction With Long-Term Results?" 2021.

29. Kamat, P.; Schweizer, R.; Kaenel, P.; Salemi, S.; Calcagni, M.; Giovanoli, P.; Gorantla, V.S.; Eberli, D.; Andres, A.-C.; Plock, J.A. Human Adipose-Derived Mesenchymal Stromal Cells May Promote Breast Cancer Progression and Metastatic Spread. Plast Reconstr Surg 2015, 136, 76-84, doi:10.1097/PRS.0000000000001321.

30. Massa, M.; Gasparini, S.; Baldelli, I.; Scarabelli, L.; Santi, P.; Quarto, R.; Repaci, E. Interaction Between Breast Cancer Cells and Adipose Tissue Cells Derived from Fat Grafting. Aesthet Surg J 2016, 36, 358-363, doi:10.1093/asj/sjv194.

31. Kronowitz, S.J.; Mandujano, C.C.; Liu, J.; Kuerer, H.M.; Smith, B.; Garvey, P.; Jagsi, R.; Hsu, L.; Hanson, S.; Valero, V. Lipofilling of the Breast Does Not Increase the Risk of Recurrence of Breast Cancer: A Matched Controlled Study. Plast Reconstr Surg 2016, 137, 385-393, doi:10.1097/01.prs.0000475741.32563.50.

32. Prantl, L.; Eigenberger, A.; Klein, S.; Limm, K.; Oefner, P.J.; Schratzenstaller, T.; Felthaus, O. Shear Force Processing of Lipoaspirates for Stem Cell Enrichment Does Not Affect Secretome of Human Cells Detected by Mass Spectrometry In Vitro. Plast Reconstr Surg 2020, 146, 749e-758e, doi:10.1097/PRS.0000000000007343. 
33. Neto, M.; Dasilva, A.; Garcia, E.; Freire, M.; Ferreira, L. Quality of Life and Self-Esteem after Breast Asymmetry Surgery. Aesthetic Surgery Journal 2007, 27, 616-621, doi:10.1016/j.asj.2007.09.002.

34. Eder, M.; Waldenfels, F. v.; Sichtermann, M.; Schuster, T.; Papadopulos, N.A.; Machens, H.-G.; Biemer, E.; Kovacs, L. ThreeDimensional Evaluation of Breast Contour and Volume Changes Following Subpectoral Augmentation Mammaplasty over 6 Months. Journal of Plastic, Reconstructive \& Aesthetic Surgery 2011, 64, 1152-1160, doi:10.1016/j.bjps.2011.03.037. 